\title{
Numerical approximation for nonlinear stochastic pantograph equations with Markovian switching
}

\author{
Shaobo Zhou* Yangzi Hu \\ School of Mathematics and Statistics, Huazhong University of Science and Technology, Wuhan 430074
}

\begin{abstract}
The main aim of the paper is to prove that the implicit numerical approximation can converge to the true solution to highly nonlinear hybrid stochastic pantograph differential equation. After providing the boundedness of the exact solution, the paper proves that the backward Euler-Maruyama numerical method can preserves boundedness of moments, and the numerical approximation converges strongly to the true solution. Finally, the exponential stability criterion on the backward EM is given, and a high order example is provided to illustrate the main result.
\end{abstract}

Key words Strong convergence; Polynomial growth conditions; Moment boundedness; Backward Euler-Maruyama method; Markovian switching; Exponential stability

\section{Introduction}

Stochastic unbounded delay systems play an important role in a variety of application areas, including biology, epidemiology, mechanic, economics and finance. The systems provide powerful models, such as, infinite delay Kolmogorov-type systems in mathematic biology [7,25], stochastic neural networks $[8,10,15]$, stochastic pantograph equations in science and engineering. So such systems have received an increasing attention $[3,6,20,21,24]$. The pantograph equation which is a very special unbounded delay equation was used by Ockendon and Taylor [18] in 1971 to study how the electric current is collected by the pantograph of an electric locomotive, from where it gets its name. Research efforts have been devoted to existence-and-uniqueness and stability of the analytical solution for stochastic pantograph equation $[1,2,16]$.

However, most of stochastic pantograph differential equations cannot be solved explicitly. Especially, explicit solutions can rarely be obtained for nonlinear stochastic pantograph equations, so numerical methods have recently received more and more attention. Fan et al. [4] investigated the convergence of the semi-implicit Euler methods for stochastic pantograph equations under the Lipschitz condition and the linear growth condition. Fan et al. [5] investigated the numerical approximation for the linear stochastic pantograph equation by using the Razumikhin technique. Milošević et al. [17] studied a Taylor polynomial approximate solution for stochastic pantograph equation with Markovian switching under the linear growth condition.

*Corresponding author. Tel.: +86 18971079628; fax: +86 27 87543231. E-mail: hustzhshbowls@sina.com(S. Zhou) 
The existing results of convergence derived by these conditions are somewhat restrictive for the purpose of practical applications, because there are many stochastic pantograph differential equations that only satisfy the local Lipschitz condition. Li et al.[9] studied convergence in probability of the Euler approximation solution for stochastic pantograph equations with Markovian switching under weaker conditions. Zhou et al.[22] studied convergence in probability of the Euler-Maruyama approximate solution of stochastic pantograph differential equation. However, to the best of the authors' knowledge, the strong convergence remains essentially unsolved for the important class of stochastic pantograph equations with super-linearly growing coefficients, owing to no result on the moment bound for the numerical solution under the polynomial growth conditions, which is the main topic of the present paper. This paper, which is natural extension of the paper [22], shall prove that a appropriate implicit approximation converges strongly to the exact solution.

This is a continuation of the first author's earlier paper [23], in which a criterion on almost surely exponential stability of numerical simulations is concerned with nonlinear stochastic pantograph equation. The paper shall develop a criterion on strong convergence of the backward Euler-Maruyama method for highly nonlinear hybrid stochastic pantograph equation. The classical linear growth condition is replaced by one-sided polynomial growth conditions, under which there exists a unique global solution and the solution is bounded. On basis of a series of lemmas, the paper shows that the backward Euler-Maruyama approximate solution converges strongly to the analytic solution. Finally, a highly nonlinear example is provided to illustrate the main theory.

The structure of the paper is as follows: In the next section, we introduce polynomial growth conditions under which stochastic pantograph systems have a unique global solutions, and prove the pth moment boundedness of the solution. Section 3 shows that the 2 nd moment of the backward EM can be bounded under one-sided polynomial growth conditions, then proposes the forward-backward EM and proves a strong convergence theorem. Section 4 gives the exponential stability criterion on the backward EM approximation. Finally, a highly nonlinear example is considered to illustrate the main theory, which implies that the results in the paper are very general, so that a wider class of nonlinear systems can satisfy these conditions.

\section{The global solution}

Throughout this paper, unless otherwise specified, let $|x|$ be the Euclidean norm in $x \in \mathbb{R}^{n}$. If $A$ is a vector or matrix, its transpose is denoted by $A^{T}$. If $A$ is a matrix, its trace norm is denoted by $|A|=\sqrt{\operatorname{trace}\left(A^{T} A\right)}$, while its operator norm is denoted by $\|A\|=\sup \{|A x|:|x|=1\}$. Let $\left(\Omega, \mathcal{F},\left\{\mathcal{F}_{t}\right\}_{t \geq 0}, \mathbb{P}\right)$ be a complete probability space with a filtration $\left\{\mathcal{F}_{t}\right\}_{t \geq 0}$, satisfying the usual conditions (i.e., it is increasing and right continuous and $\mathcal{F}_{0}$ contains all P-null sets). Let $\mathbb{R}_{+}=[0,+\infty)$. Let $w(t)=\left(w_{1}(t), \cdots \cdot, w_{m}(t)\right)$ be an $\bar{m}$-dimensional Brownian motion.

Let $r(t)(t \geq 0)$ be a right-continuous Markov chain on the probability space taking values in 
a finite state space $S=\{1,2, \ldots, N\}$ with generator $\Gamma=\left(\gamma_{i j}\right)_{N \times N}$ given by

$$
P\{r(t+\Delta)=j \mid r(t)=i\}=\left\{\begin{array}{cl}
\gamma_{i j} \Delta+0(\Delta), & \text { if } i \neq j \\
1+\gamma_{i i} \Delta+0(\Delta), & \text { if } i=j
\end{array}\right.
$$

where $\Delta>0$. Here $\gamma_{i j} \geq 0$ is the transition rate from $i$ to $j$ if $i \neq j$, while $\gamma_{i i}=-\sum_{j \neq i} \gamma_{i j}$.

Consider an n-dimensional stochastic pantograph equation with Markovian switching

$$
\mathrm{d} x(t)=f(x(t), x(q t), r(t)) \mathrm{d} t+g(x(t), x(q t), r(t)) \mathrm{d} w(t), \quad 0<q<1
$$

on $t \geq 0$ with the initial data $x_{0} \in \mathbb{R}^{n}, f: \mathbb{R}^{n} \times \mathbb{R}^{n} \longrightarrow \mathbb{R}^{n}, g: \mathbb{R}^{n} \times \mathbb{R}^{n} \longrightarrow \mathbb{R}^{n}$ are locally Lipschitz continuous.

(H1) (The Local Lipschitz Condition) For each integer $R \geq 1$, there exists a positive constant $C_{R}$ such that

$$
\left|f\left(x_{1}, y_{1}, i\right)-f\left(x_{2}, y_{2}, i\right)\right| \vee\left|g\left(x_{1}, y_{1}, i\right)-g\left(x_{2}, y_{2}, i\right)\right| \leq C_{R}\left(\left|x_{1}-x_{2}\right|+\left|y_{1}-y_{2}\right|\right)
$$

for all $i \in \mathbb{S}$ and $x_{k}, y_{k} \in \mathbb{R}^{n}$ with $\left|x_{k}\right| \vee\left|y_{k}\right| \leq R(k=1,2)$.

(H2) (Polynomial growth conditions) For all $i \in S$ and any $x \in \mathbb{R}^{n}$, there exist positive constants $\alpha, a, \bar{a}, a_{0}, b, \bar{b}, b_{0}$ such that

$$
\begin{gathered}
2\langle x(s), f(x(s), x(q s), i)\rangle \leq-a|x(s)|^{\alpha+2}+\bar{a}|x(q s)|^{\alpha+2}-a_{0}|x(s)|^{2}, \\
|g(x(s), x(q s), i)|^{2} \leq b|x(s)|^{\alpha+2}+\bar{b}|x(q s)|^{\alpha+2}+b_{0}|x(s)|^{2} .
\end{gathered}
$$

Theorem 2.1 For $p \geq 2$, let (H1) and (H2) hold with $a_{0}>(p-1) b_{0}, a>(p-1) b+\frac{p-2+q^{-1}(\alpha+2)}{\alpha+p}(\bar{a}+$ $(p-1) \bar{b})$. Then for any initial data $x_{0}$, there almost surely exists a unique global solution $x(t)$ to Eq.(2.1) on $t \geq-\tau$. Moreover,

$$
\mathbb{E}|x(t)|^{p} \leq \mathbb{E}\left|x_{0}\right|^{p}
$$

Proof Under (H1), applying the standing truncation technique [see Mao[11], Theorem 3.2.2, $\left.P_{95}\right]$ to Eq.(2.1) for any initial data $\xi$, there exists a unique maximal local strong solution to Eq.(2.1) on $-\tau<t<\nu_{e}$, where $\nu_{e}$ is the explosion time. To show this solution is global, we only need to show that $\nu_{e}=\infty$ a.s. Let $k_{0}>0$ be sufficiently large such that $k_{0}>\left|x_{0}\right|$. For each integer $k \geq k_{0}$, define the stopping time

$$
\nu_{k}=\inf \left\{t \in\left[0, \nu_{e}\right):|x(t)| \geq k\right\},(k \in N),
$$

where throughout this paper, we set $\inf \emptyset=\infty$ (as usual, $\emptyset=$ the empty set). By the definition of the stopping time $\nu_{k}$, it is obvious that $\nu_{k}$ is an increasing function with $k$, so $\nu_{k} \rightarrow \nu_{\infty} \leq \nu_{e}(k \rightarrow$ $\infty) a . s$. If we can show that $\nu_{\infty}=\infty$ a.s., then $\nu_{e}=\infty$ a.s. which implies that $x(t)$ is global. In other words, we only prove that $\mathbb{P}\left(\nu_{k} \leq t\right) \rightarrow 0(k \rightarrow \infty, t>0)$. Define $V(x)=|x|^{p}$, since $P\left(\nu_{k} \leq t\right) V\left(x\left(\nu_{k}\right)\right) \leq \mathbb{E} V\left(x\left(t \wedge \nu_{k}\right)\right)$, that is, we only need to prove that $\mathbb{E} V\left(x\left(t \wedge \nu_{k}\right)\right)<+\infty$ since $V\left(x\left(\nu_{k}\right)\right)=\left|x\left(\nu_{k}\right)\right|^{p}=k^{p} \rightarrow \infty$. By the Itô formula, we obtain

$$
V(x(t))=V(\xi(0))+\int_{0}^{t} L V(x(s), x(q s), r(s)) \mathrm{d} s+\int_{0}^{t} V_{x}(x(s)) g(x(s), x(q s), r(s)) \mathrm{d} w(s),
$$


where

$L V(x(s), x(q s), r(s)) \leq p|x(s)|^{p-2} x^{T}(s) f(x(s), x(q s), r(s))+\frac{p(p-1)}{2}|x(s)|^{p-2}|g(x(s), x(q s), r(s))|^{2}$.

Making use of (H2), one may compute

$$
\begin{aligned}
L V(x(s), x(q s), r(s)) \leq & \frac{p}{2}|x(s)|^{p-2}\left[-a|x(s)|^{\alpha+2}+\bar{a}|x(q s)|^{\alpha+2}-a_{0}|x(s)|^{2}\right] \\
& +\left.\frac{p(p-1)}{2}|x(s)|\right|^{p-2}\left[b|x(s)|^{\alpha+2}+\bar{b}|x(q s)|^{\alpha+2}+b_{0}|x(s)|^{2}\right] \\
\leq & \frac{p}{2}|x(s)|^{p-2}\left[-\left(a_{0}-(p-1) b_{0}\right)|x(s)|^{2}-(a-(p-1) b)|x(s)|^{\alpha+2}\right] \\
& +\frac{p}{2}(\bar{a}+(p-1) \bar{b})|x(s)|^{p-2}|x(q s)|^{\alpha+2} .
\end{aligned}
$$

The inequality $a^{p} b^{q} \leq \frac{p}{p+q} a^{p+q}+\frac{q}{p+q} b^{p+q}, a, b, p, q>0$, implies

$$
\begin{aligned}
|x(s)|^{p-2}|x(q s)|^{\alpha+2} & \leq \frac{p-2}{\alpha+p}|x(s)|^{\alpha+p}+\frac{\alpha+2}{\alpha+p}|x(q s)|^{\alpha+p} \\
& =\frac{\alpha+2}{\alpha+p}\left(|x(q s)|^{\alpha+p}-\frac{1}{q}|x(s)|^{\alpha+p}\right)+\frac{p-2+q^{-1}(\alpha+2)}{\alpha+p}|x(s)|^{\alpha+p} .
\end{aligned}
$$

Substituting (2.4) into (2.3), yields

$$
\begin{aligned}
L V(x(s), x(q s)) \leq & \frac{p}{2}|x(s)|^{p-2}\left[-\left(a_{0}-(p-1) b_{0}\right)|x(s)|^{2}-(a-(p-1) b)|x(s)|^{\alpha+2}\right] \\
& +\frac{p}{2}(\bar{a}+(p-1) \bar{b})\left[\frac{\alpha+2}{\alpha+p}\left(|x(q s)|^{\alpha+p}-\frac{1}{q}|x(s)|^{\alpha+p}\right)+\frac{p-2+q^{-1}(\alpha+2)}{\alpha+p}|x(s)|^{\alpha+p}\right] \\
\leq & \frac{p(\alpha+2)}{2(\alpha+p)}(\bar{a}+(p-1) \bar{b})\left(|x(q s)|^{\alpha+p}-\frac{1}{q}|x(s)|^{\alpha+p}\right)-I_{0}(x),
\end{aligned}
$$

where

$I_{0}(x(s))=\frac{p}{2}\left(a_{0}-(p-1) b_{0}\right)|x(s)|^{p}+\frac{p}{2}\left[a-(p-1) b-\frac{p-2+q^{-1}(\alpha+2)}{\alpha+p}(\bar{a}+(p-1) \bar{b})\right]|x(s)|^{\alpha+p}$

Recalling that $a_{0}>(p-1) b_{0}, a>(p-1) b+\frac{p-2+q^{-1}(\alpha+2)}{\alpha+p}(\bar{a}+(p-1) \bar{b})$, there exists a positive constant $c_{0}$ such that

$$
I_{0}(x(s)) \geq c_{0}|x(s)|^{p}
$$

Substituting this into (2.5), (2.5) into (2.2), yields

$$
\begin{aligned}
V(x(t)) \leq & V(\xi(0)))+\frac{p}{2} \frac{\alpha+2}{\alpha+p}(\bar{a}+(p-1) \bar{b}) \int_{0}^{t}\left(|x(q s)|^{\alpha+p}-\frac{1}{q}|x(s)|^{\alpha+p}\right) \mathrm{d} s \\
& -c_{0} \int_{0}^{t}|x(s)|^{p} \mathrm{~d} s+\int_{0}^{t} V_{x}(x(s)) g(x(s), x(q s), r(s)) \mathrm{d} w(s) .
\end{aligned}
$$

Applying the substitution technique of integral, one may get

$$
\int_{0}^{t}\left(|x(q s)|^{\alpha+p}-\frac{1}{q}|x(s)|^{\alpha+p}\right) \mathrm{d} s \leq \frac{1}{q} \int_{0}^{q t}|x(s)|^{\alpha+p} \mathrm{~d} s-\frac{1}{q} \int_{0}^{t}|x(s)|^{\alpha+p} \mathrm{~d} s \leq-\frac{1}{q} \int_{q t}^{t}|x(s)|^{\alpha+p} \mathrm{~d} s .
$$

Substituting (2.7) into (2.6), taking expectation and noting that $\int_{0}^{t \wedge \nu_{k}} V_{x}(x(s)) g(x(s), x(q s), r(s)) \mathrm{d} w(s)$ is local martingale, so we have

$$
\mathbb{E} V\left(x\left(t \wedge \nu_{k}\right)\right) \leq \mathbb{E} V(\xi)-\frac{p(\alpha+2)}{2 q(\alpha+p)}(\bar{a}+(p-1) \bar{b}) \mathbb{E} \int_{q t}^{t}|x(s)|^{\alpha+p} \mathrm{~d} s-c_{0} \mathbb{E} \int_{0}^{t}|x(s)|^{p} \mathrm{~d} s \leq \mathbb{E}\left|x_{0}\right|^{p} .
$$

Letting $k \rightarrow \infty$, which implies the required result. 


\section{Strong convergence}

In the section, our main objective is to prove that the numerical approximation of Eq.(2.1) strongly converges to the true solution. After introducing the backward EM scheme and its continuous form of numerical method, we prove the pth moment bound and the main strong convergence theorem is proved.

Given a step size $\Delta \in(0,1)$, and for $t \in[0, T], M \Delta=T$ for some positive integer $M$. Let $t_{k}=k \Delta(k \geq 0),[t / \Delta]$ be the integer part of $t / \Delta$.

Lemma 3.1(see [30]) Let $r_{k}^{\Delta}=r\left(t_{k}\right)$ for $\Delta>0$ and $k \geq 0$, then $\left\{r_{k}^{\Delta}, k=0,1,2, \ldots\right\}$ is discrete Markovian chain with the one-step transition probability matrix

$$
P(\Delta)=\left(P_{i j}(\Delta)\right)_{N \times N}=\mathrm{e}^{\Delta \Gamma} .
$$

Since $\gamma_{i j}$ are independent of $x$, the paths of $r$ can be generated independently of $x$ and, in fact, before computing $x$.

The discrete Markov chain $\left\{r_{k}^{\Delta}, k=0,1,2, \ldots\right\}$ can be simulated as follows: Compute the one-step transition probability matrix $P(\Delta)$. Let $r_{0}^{\Delta}=i_{0}$ and generate a random number $\xi_{1}$ which is uniformly distributed in $[0,1]$. Define

$$
r_{1}^{\Delta}=\left\{\begin{array}{cc}
i_{1} & \text { if } i_{1} \in S-\{N\} \text { such that } \sum_{j=1}^{i_{1}-1} P_{i_{0}, j}(\Delta) \leq \xi_{1}<\sum_{j=1}^{i_{1}} P_{i_{0}, j}(\Delta), \\
N, & \text { if } \sum_{j=1}^{N-1} P_{i_{0}, j}(\Delta) \leq \xi_{1},
\end{array}\right.
$$

where we set $\sum_{j=1}^{0} P_{i_{0}, j}(\Delta)=0$ as usual.Generate independently a new random number $\xi_{2}$ which is again uniformly distributed in $[0,1]$ and then define

$$
r_{2}^{\Delta}=\left\{\begin{array}{cc}
i_{2} & \text { if } i_{2} \in S-\{N\} \text { such that } \sum_{j=1}^{i_{2}-1} P_{r_{1}^{\Delta}, j}(\Delta) \leq \xi_{2}<\sum_{j=1}^{i_{2}} P_{r_{1}^{\Delta}, j}(\Delta), \\
N, & \text { if } \sum_{j=1}^{N-1} P_{r_{1}^{\Delta}, j}(\Delta) \leq \xi_{2} .
\end{array}\right.
$$

Repeating this procedure a trajectory of $\left\{r_{k}^{\Delta}, k=1,2, \ldots\right\}$ can be generated. This procedure can be carried out independently to obtain more trajectories. After explaining how to simulate the discrete Markovian chain $\left\{r_{k}^{\Delta}, k=0,1,2, \ldots\right\}$, we can now define the backward Euler-Maruyama approximate solution for Eq.(2.1). Compute the discrete approximations $X\left(t_{k}\right) \approx x\left(t_{k}\right)$ by setting $X_{0}=x_{0}, r_{0}^{\Delta}=i_{0}$ and performing

$$
X\left(t_{k+1}\right)=X\left(t_{k}\right)+f\left(X\left(t_{k+1}\right), X\left(t_{[q(k+1)]}\right), r\left(t_{k+1}\right)\right) \Delta+g\left(X\left(t_{k}\right), X\left(t_{[q k]}\right), r\left(t_{k}\right)\right) \Delta w\left(t_{k}\right) .
$$

Let $t_{k}=k \Delta(k \geq 0) . X\left(t_{k}\right)$ is an approximation to $x\left(t_{k}\right)$. Moreover, the increments $\Delta w_{t_{k}}=$ $w\left(t_{k+1}\right)-w\left(t_{k}\right), k=1,2, \ldots$, are independent $N(0, \Delta)$-distributed Gaussian random variables $\mathcal{F}_{t_{k}}$ measurable at the mesh-point $t_{k}$. Once we compute the discrete values $X\left(t_{k}\right)$ from the backward EM scheme (3.1), then we define the discrete forward backward EM scheme ( see $[13,14,19])$

$$
\hat{X}\left(t_{k+1}\right)=\hat{X}\left(t_{k}\right)+f\left(X\left(t_{k}\right), X\left(t_{[q k]}\right), r\left(t_{k}\right)\right) \Delta+g\left(X\left(t_{k}\right), X\left(t_{[q k]}\right), r\left(t_{k}\right)\right) \Delta w_{t_{k}},
$$

where $\hat{X}(0)=X(0)=x_{0}$. Define

$$
\eta(t)=t_{k}, \quad t \in\left[t_{k}, t_{k+1}\right), \quad k \geq 0 .
$$


The continuous forward backward EM scheme is defined by

$\bar{X}(t)=\bar{X}(0)+\int_{0}^{t} f(X(\eta(s)), X(\eta(q s)), r(\eta(s))) \mathrm{d} s+\int_{0}^{t} g(X(\eta(s)), X(\eta(q s)), r(\eta(s))) \mathrm{d} w(s), \quad t \geq 0$.

Note that the continuous and discrete forward backward EM scheme coincide at the grid point, $\bar{X}\left(t_{k}\right)=\hat{X}\left(t_{k}\right)$.

To guarantee that this method (3.1) is well defined, we impose the following one-sided Lipschitz condition on the drift coefficient $f(x, y, i)$.

(H3) ( One-sided Lipschitz condition) There exists a positive constant $\lambda>0$ such that for any $x_{i} \in \mathbb{R}^{n}(i=1,2)$

$$
\left\langle x_{1}-x_{2}, f\left(x_{1}, y, i\right)-f\left(x_{2}, y, i\right)\right\rangle \leq \lambda\left|x_{1}-x_{2}\right|^{2} .
$$

Applying a fixed point theorem one can prove that Eq.(3.1) has a unique solution $X\left(t_{k+1}\right)$ given $X\left(t_{k}\right)$ if $\lambda \Delta<1$, then the backward EM scheme (3.1) is well defined ( see e.g.[12]). From now on we always assume that $\Delta<\lambda^{-1}$.

For convenience, let integer $R>0$ be arbitrary and define the sequence of stopping time

$$
\sigma_{R}=\inf \left\{k:\left|X\left(t_{k}\right)\right|>R\right\}
$$

Clearly, when $k \in\left[0, \sigma_{R}(w)\right],\left|X\left(t_{i}\right)(w)\right| \leq R, i=1, \cdots, k-1$, but we may have $\left|X\left(t_{k}\right)(w)\right|>R$.

Lemma 3.1 Let (H1),(H2) and (H3) hold. Then for any $p \geq 2$ and sufficiently large integer $R$, there exists a constant $C(R, p)$ such that

$$
\mathbb{E}\left[\left|X\left(t_{k}\right)\right|^{p} I_{\left[0, \sigma_{R}\right]}(k)\right]<C(R, p), \forall k \geq 0
$$

Proof By the inequality $\langle a, b\rangle \leq|a||b| \leq \frac{1}{2}\left(|a|^{2}+|b|^{2}\right)$, one may get

$$
\begin{aligned}
\left|X\left(t_{k}\right)\right|^{2}= & \left\langle X\left(t_{k}\right), X\left(t_{k-1}\right)+f\left(X\left(t_{k}\right), X\left(t_{[q k]}\right), r\left(t_{k}\right)\right) \Delta+g\left(X\left(t_{k-1}\right), X\left(t_{[q(k-1)]}\right), r\left(t_{k-1}\right)\right) \Delta w_{t_{k-1}}\right\rangle \\
= & \left\langle X\left(t_{k}\right), f\left(X\left(t_{k}\right), X\left(t_{[q k]}\right), r\left(t_{k}\right)\right)\right\rangle \Delta+\left\langle X\left(t_{k}\right), X\left(t_{k-1}\right)\right. \\
& \left.+g\left(X\left(t_{k-1}\right), X\left(t_{[q(k-1)]}\right), r\left(t_{k-1}\right)\right) \Delta w_{t_{k-1}}\right\rangle \\
\leq \quad & -\frac{a}{2}\left|X\left(t_{k}\right)\right|^{\alpha+2} \Delta+\frac{\bar{a}}{2}\left|X\left(t_{[q k]}\right)\right|^{\alpha+2} \Delta-\frac{a_{0}}{2}\left|X\left(t_{k}\right)\right|^{2} \Delta+\frac{1}{2}\left|X\left(t_{k}\right)\right|^{2} \\
& +\frac{1}{2}\left|X\left(t_{k-1}\right)+g\left(X\left(t_{k-1}\right), X\left(t_{[q(k-1)]}\right), r\left(t_{k-1}\right)\right) \Delta w_{t_{k-1}}\right|^{2}
\end{aligned}
$$

That is,

$$
\left|X\left(t_{k}\right)\right|^{2} \leq \bar{a}\left|X\left(t_{[q k]}\right)\right|^{\alpha+2} \Delta+\left|X\left(t_{k-1}\right)+g\left(X\left(t_{k-1}\right), X\left(t_{[q(k-1)]}\right), r\left(t_{k-1}\right)\right) \Delta w_{t_{k-1}}\right|^{2}
$$

Applying inequality $|a+b+c|^{\frac{p}{2}} \leq 3^{\frac{p}{2}-1}\left(a^{\frac{p}{2}}+b^{\frac{p}{2}}+c^{\frac{p}{2}}\right), \quad a, b, c>0$, yields

$$
\left|X\left(t_{k}\right)\right|^{p} \leq 3^{\frac{p}{2}-1}\left[(\bar{a} \Delta)^{\frac{p}{2}}\left|X\left(t_{[q k]}\right)\right|^{\frac{p(\alpha+2)}{2}}+2^{\frac{p}{2}}\left|X\left(t_{k-1}\right)\right|^{p}+2^{\frac{p}{2}}\left|g\left(X\left(t_{k-1}\right), X\left(t_{[q(k-1)]}\right), r\left(t_{k-1}\right)\right) \Delta w_{t_{k-1}}\right|^{p}\right] .
$$

Hence

$$
\begin{aligned}
\mathbb{E}\left[\left|X\left(t_{k}\right)\right|^{p} \mathbf{1}_{\left[0, \sigma_{R}\right]}(k)\right] \leq & 3^{\frac{p}{2}-1}\left\{(\bar{a} \Delta)^{\frac{p}{2}} \mathbb{E}\left[\left|X\left(t_{[q k]}\right)\right|^{\frac{p(\alpha+2)}{2}} 1_{\left[0, \sigma_{R}\right]}(k)\right]+2^{\frac{p}{2}} \mathbb{E}\left[\left|X\left(t_{k-1}\right)\right|^{p} 1_{\left[0, \sigma_{R}\right]}(k)\right]\right. \\
& \left.+2^{\frac{p}{2}} \mathbb{E}\left[\left|g\left(X\left(t_{k-1}\right), X\left(t_{[q(k-1)]}\right), r\left(t_{k-1}\right)\right) \Delta w_{t_{k-1}}\right|^{p} 1_{\left[0, \sigma_{R}\right]}(k)\right]\right\}
\end{aligned}
$$


It is easy to obtain that $\left|g\left(X\left(t_{k-1}\right), X\left(t_{[q(k-1)]}\right)\right)\right|,\left|X\left(t_{[q k]}\right)\right|,\left|X\left(t_{k-1}\right)\right|$ are bound for $\left|X\left(t_{i}\right)(\omega)\right|<$ $R, i \leq k-1$ if $k \in\left[0, \sigma_{R}(\omega)\right]$. By the Hölder inequality, then we may compute

$$
\begin{aligned}
& \mathbb{E}\left[\left|g\left(X\left(t_{k-1}\right), X\left(t_{[q(k-1)]}\right)\right) \Delta w_{t_{k-1}}\right|^{p} \mathbf{1}_{\left[0, \sigma_{R}\right]}(k)\right] \\
\leq & \left(\mathbb{E}\left[\left|g\left(X\left(t_{k-1}\right), X\left(t_{[q(k-1)]}\right), r\left(t_{k-1}\right)\right)\right|^{2 p} 1_{\left[0, \sigma_{R}\right]}(k)\right]\right)^{\frac{1}{2}}\left(\mathbb{E}\left|\Delta w_{t_{k-1}}\right|^{2 p}\right)^{\frac{1}{2}} .
\end{aligned}
$$

According to the property of normal distributed, and noting that $\Delta w_{t_{k-1}}$ is $\bar{m}$-dimensional Brownian motion, it is easy to see that there exists a constant $c(p)$ such that

$$
\mathbb{E}\left|\Delta w_{t_{k-1}}\right|^{2 p}=c(p) \Delta^{p} .
$$

Substituting this into (3.9), then there exists a positive constant $C(R, p)$ such that

$$
\mathbb{E}\left[\left|g\left(X\left(t_{k-1}\right), X\left(t_{[q(k-1)]}\right), r\left(t_{k-1}\right)\right) \Delta w_{t_{k-1}}\right|^{p} 1_{\left[0, \sigma_{R}\right]}(k)\right] \leq C(R, p) \Delta^{\frac{p}{2}} .
$$

Substituting for this into (3.8) yields

$$
\mathbb{E}\left[\left|X\left(t_{k}\right)\right|^{p} \mathbf{1}_{\left[0, \sigma_{R}\right]}(k)\right] \leq C(R, p) .
$$

To avoid complicate notations, the product of $C(R, p)$ and another constant is still denoted by $C(R, p)$. In the following, other constants is similar.

Lemma 3.2 Let (H1),(H2) and (H3) hold with $a_{0}>m b_{0}, a>\bar{a}+m b+m \bar{b}$. Then

$$
\mathbb{E}\left|X\left(t_{k}\right)\right|^{2} \leq\left(1+a_{0} \Delta\right) \mathbb{E}\left|X_{0}\right|^{2}+m b\left|X_{0}\right|^{\alpha+2} \Delta .
$$

Proof By (3.1) and (H2), we have

$$
\begin{aligned}
\left|X\left(t_{k+1}\right)\right|^{2} \leq & -\frac{a}{2}\left|X\left(t_{k+1}\right)\right|^{\alpha+2} \Delta+\frac{\bar{a}}{2}\left|X\left(t_{[q(k+1)]}\right)\right|^{\alpha+2} \Delta \frac{a_{0}}{2}\left|X\left(t_{k+1}\right)\right|^{2} \Delta \\
& +\frac{1}{2}\left|X\left(t_{k+1}\right)\right|^{2}+\frac{1}{2} \mid X\left(t_{k}\right)+g\left(X\left(t_{k}\right),\left.X\left(t_{[q k]}, r\left(t_{k}\right)\right) \Delta w_{t_{k}}\right|^{2}\right. \\
\leq & -\left.\frac{a}{2}\left|X\left(t_{k+1}\right)\right|\right|^{\alpha+2} \Delta+\frac{\bar{a}}{2}\left|X\left(t_{[q(k+1)]}\right)\right|^{\alpha+2} \Delta-\frac{a_{0}}{2}\left|X\left(t_{k+1}\right)\right|^{2} \Delta \\
& +\frac{1}{2}\left|X\left(t_{k+1}\right)\right|^{2}+\frac{1}{2}\left|g\left(X\left(t_{k}\right), X\left(t_{[q k]}\right), r\left(t_{k}\right)\right) \Delta w_{t_{k}}\right|^{2} \\
& +\frac{1}{2}\left|X\left(t_{k}\right)\right|^{2}+\left\langle X\left(t_{k}\right), g\left(X\left(t_{k}\right), X\left(t_{[q k]}\right), r\left(t_{k}\right)\right) \Delta w_{t_{k}}\right\rangle .
\end{aligned}
$$

Then

$$
\begin{aligned}
\left(1+a_{0} \Delta\right)\left|X\left(t_{k+1}\right)\right|^{2} \leq & -a\left|X\left(t_{k+1}\right)\right|^{\alpha+2} \Delta+\bar{a}\left|X\left(t_{[q(k+1)]}\right)\right|^{\alpha+2} \Delta+\left|X\left(t_{k}\right)\right|^{2} \\
& +m\left(b\left|X\left(t_{k}\right)\right|^{\alpha+2}+\bar{b}\left|X\left(t_{[q k]}\right)\right|^{\alpha+2}+b_{0}\left|X\left(t_{k}\right)\right|^{2}\right) \Delta+s_{k} .
\end{aligned}
$$

where

$$
\left.s_{k}=\left|g\left(X\left(t_{k}\right), X\left(t_{[q k]}\right)\right)\right|^{2}\left(\left|\Delta w_{t_{k}}\right|^{2}-m \Delta\right)+2\left\langle X\left(t_{k}\right), g\left(X\left(t_{k}\right), X\left(t_{[q k]}\right)\right), r\left(t_{k}\right)\right) \Delta w_{t_{k}}\right\rangle
$$

Then

$$
\begin{aligned}
\left(1+a_{0} \Delta\right)\left(\left|X\left(t_{k+1}\right)\right|^{2}-\left|X\left(t_{k}\right)\right|^{2}\right) \leq & \left(m b_{0}-a_{0}\right) \Delta\left|X\left(t_{k}\right)\right|^{2}+\bar{a}\left|X\left(t_{[q(k+1)]}\right)\right|^{\alpha+2} \Delta-a\left|X\left(t_{k+1}\right)\right|^{\alpha+2} \Delta \\
& +m\left(b\left|X\left(t_{k}\right)\right|^{\alpha+2}+\bar{b}\left|X\left(t_{[q k]}\right)\right|^{\alpha+2}\right) \Delta+s_{k} .
\end{aligned}
$$


Summing up both sides of inequality (3.10) from 0 to $k-1$, yields

$$
\begin{aligned}
\left(1+a_{0} \Delta\right)\left|X\left(t_{k}\right)\right|^{2} \leq & \left(1+a_{0} \Delta\right)\left|X\left(t_{0}\right)\right|^{2}+\left(m b_{0}-a_{0}\right) \Delta \sum_{i=0}^{k-1}\left|X\left(t_{i}\right)\right|^{2}+\bar{a} \sum_{i=0}^{k-1}\left|X\left(t_{[q(i+1)]}\right)\right|^{\alpha+2} \Delta \\
& -a \sum_{i=0}^{k-1}\left|X\left(t_{i+1}\right)\right|^{\alpha+2} \Delta+m \sum_{i=0}^{k-1}\left(b\left|X\left(t_{i}\right)\right|^{\alpha+2}+\bar{b}\left|X\left(t_{[q i}\right)\right|^{\alpha+2}\right) \Delta+S_{k} .
\end{aligned}
$$

where $S_{k}=\sum_{i=0}^{k-1} s_{i}$. Noting that

$$
\sum_{i=0}^{k-1}\left|X\left(t_{[q i]}\right)\right|^{\alpha+2} \leq \sum_{i=0}^{[q(k-1)]+1}\left|X\left(t_{i}\right)\right|^{\alpha+2} .
$$

Similarly,

$$
\sum_{i=0}^{k-1}\left|X\left(t_{[q(i+1)]}\right)\right|^{\alpha+2}=\sum_{i=1}^{k}\left|X\left(t_{[q i]}\right)\right|^{\alpha+2} \leq \sum_{i=0}^{[q k]+1}\left|X\left(t_{i}\right)\right|^{\alpha+2} .
$$

Substituting these into (3.11), yields

$$
\begin{aligned}
\left|X\left(t_{k}\right)\right|^{2} \leq & \left(1+a_{0} \Delta\right)\left|X\left(t_{0}\right)\right|^{2}+\left(m b_{0}-a_{0}\right) \sum_{i=0}^{k-1}\left|X\left(t_{i}\right)\right|^{2} \Delta+\bar{a} \sum_{i=0}^{[q k]+1}\left|X\left(t_{i}\right)\right|^{\alpha+2} \Delta \\
& -a \sum_{i=1}^{k}\left|X\left(t_{i}\right)\right|^{\alpha+2} \Delta+m b \sum_{i=0}^{k-1}\left|X\left(t_{i}\right)\right|^{\alpha+2} \Delta+m \bar{b} \sum_{i=0}^{[q(k-1)]+1}\left|X\left(t_{i}\right)\right|^{\alpha+2} \Delta+S_{k} .
\end{aligned}
$$

Replacing $k$ by $k \wedge \sigma_{R}$ and taking expectation on two sides of the above inequality, yields

$$
\begin{aligned}
\mathbb{E}\left|X\left(t_{k \wedge \sigma_{R}}\right)\right|^{2} \leq & \left(1+a_{0} \Delta\right) \mathbb{E}\left|X\left(t_{0}\right)\right|^{2}+\left(m b_{0}-a_{0}\right) \Delta \sum_{i=0}^{k-1} \mathbb{E}\left|X\left(t_{i \wedge \sigma_{R}}\right)\right|^{2} \\
& +\bar{a} \sum_{i=0}^{[q k]+1} \mathbb{E}\left|X\left(t_{i \wedge \sigma_{R}}\right)\right|^{\alpha+2} \Delta-a \sum_{i=1}^{k} \mathbb{E}\left|X\left(t_{i \wedge \sigma_{R}}\right)\right|^{\alpha+2} \Delta \\
& +m b \sum_{i=0}^{k-1} \mathbb{E}\left|X\left(t_{i \wedge \sigma_{R}}\right)\right|^{\alpha+2} \Delta+m \bar{b} \sum_{i=0}^{[q(k-1)]+1} \mathbb{E}\left|X\left(t_{i \wedge \sigma_{R}}\right)\right|^{\alpha+2} \Delta+\mathbb{E} S_{k \wedge \sigma_{R}} .
\end{aligned}
$$

Noting that $S_{k \wedge \sigma_{R}}$ is local martingale by Lemma 3.1, and recalling that $a_{0}>m b_{0}, a>a_{d}+$ $m b_{d}+m b$, then

$$
\begin{aligned}
\mathbb{E}\left|X\left(t_{k \wedge \sigma_{R}}\right)\right|^{2} \leq & \left(1+a_{0} \Delta\right) \mathbb{E}\left|X\left(t_{0}\right)\right|^{2}-\left(a_{0}-m b_{0}\right) \sum_{i=0}^{k-1} \mathbb{E}\left|X\left(t_{i \wedge \sigma_{R}}\right)\right|^{2} \\
& -(a-\bar{a}-m \bar{b}-m b) \sum_{i=1}^{k} \mathbb{E}\left|X\left(t_{i \wedge \sigma_{R}}\right)\right|^{\alpha+2} \Delta+m b\left|X\left(t_{0}\right)\right|^{\alpha+2} \Delta+\mathbb{E} S_{k \wedge \sigma_{R}} \\
\leq & \left(1+a_{0} \Delta\right) \mathbb{E}\left|X_{0}\right|^{2}+m b\left|X_{0}\right|^{\alpha+2} \Delta+\mathbb{E} S_{k \wedge \sigma_{R}},
\end{aligned}
$$

noting that the right bound is independent of $R$. Therefore, letting $R \rightarrow \infty$ and applying the Fatou's lemma, there exists a positive constant such that

$$
\mathbb{E}\left|X\left(t_{k}\right)\right|^{2} \leq\left(1+a_{0} \Delta\right) \mathbb{E}\left|X_{0}\right|^{2}+m b\left|X_{0}\right|^{\alpha+2} \Delta .
$$

The proof is complete.

Lemma 3.3 Let (H1), (H2) and (H3) hold, and assume that for any $x, y \in \mathbb{R}^{n}$

$$
|f(x, y)| \leq c|x|+\bar{c}|y|^{\alpha+1}+c|x|^{\alpha+1} .
$$


Then, for any integer $p \geq 2$, there exists a positive constant $C(R, p)$ such that

$$
\mathbb{E}\left[\left|\hat{X}\left(t_{k}\right)-X\left(t_{k}\right)\right|^{p} 1_{\left[0, \sigma_{R}\right]}(k)\right] \leq C(R, p) \Delta^{p}, \quad \forall k \in \mathbb{N} .
$$

Proof Summing up both schemes of the backward EM and forward backward EM, we have

$$
\begin{aligned}
\hat{X}\left(t_{k+1}\right)-X\left(t_{k+1}\right)= & \hat{X}\left(t_{k}\right)-X\left(t_{k}\right)+\left[f\left(X\left(t_{k}\right), X\left(t_{[q k]}\right), r\left(t_{k}\right)\right)\right. \\
& -f\left(X\left(t_{k+1}\right), X\left(t_{[q(k+1)]}\right), r\left(t_{k+1}\right)\right) \Delta .
\end{aligned}
$$

Summing up the above equality from 0 to $k$, yields

$$
\hat{X}\left(t_{k}\right)-X\left(t_{k}\right)=\left(f\left(X_{0}, X_{0}, r_{0}\right)-f\left(X\left(t_{k}\right), X\left(t_{[q k]}\right), r\left(t_{k}\right)\right) \Delta .\right.
$$

Applying the Hölder inequality, Lemma 3.1 and (3.12), we have

$$
\begin{aligned}
\mathbb{E}\left[\left|\hat{X}\left(t_{k}\right)-X\left(t_{k}\right)\right|^{p} \mathbf{1}_{\left[0, \sigma_{R}\right]}(k)\right] & \leq \mathbb{E}\left[\left|f\left(X_{0}, X_{0}, r_{0}\right)-f\left(X\left(t_{k}\right), X\left(t_{[q k]}\right), r\left(t_{k}\right)\right)\right|^{p} \mathbf{1}_{\left[0, \sigma_{R}\right]}(k)\right] \Delta^{p} \\
& \leq C(R, p) \Delta^{p} .
\end{aligned}
$$

Theorem 3.4 Assume that (H1),(H2) and (H3) hold with $a_{0}>m b_{0}, a>m b+(\bar{a}+m \bar{b}) / q$. Then for any given $\varepsilon>0$, there exists a sufficiently large positive integer $R_{0}$ and sufficiently small $\Delta^{*}$ such that for every $R>R_{0}$ and $\Delta<\Delta^{*}$

$$
P\left(\rho_{R} \leq T\right) \leq \varepsilon, \quad T>0
$$

where $\rho_{R}=\inf \{t>0,|\bar{X}(t)| \geq R$ or $|X(\eta(t))|>R\}$.

Proof By the Itô formula, one may compute

$$
\begin{aligned}
\left|\bar{X}\left(T \wedge \rho_{R}\right)\right|^{2}= & |\bar{X}(0)|^{2}+\int_{0}^{T \wedge \rho_{R}}(2\langle\bar{X}(s), f(X(\eta(s)), X(\eta(q s)), r(\eta(s)))\rangle \\
& \left.+|g(X(\eta(s)), X(\eta(q s)), r(\eta(s)))|^{2}\right) \mathrm{d} s \\
& +2 \int_{0}^{T \wedge \rho_{R}}\langle\bar{X}(s), g(X(\eta(s)), X(\eta(q s)), r(\eta(s)))\rangle \mathrm{d} w(s) \\
\leq \quad & |\bar{X}(0)|^{2}+\int_{0}^{T \wedge \rho_{R}}(2\langle X(\eta(s)), f(X(\eta(s)), X(\eta(q s)), r(\eta(s)))\rangle \\
& \left.+|g(X(\eta(s)), X(\eta(q s)), r(\eta(s)))|^{2}\right) \mathrm{d} s \\
& +2 \int_{0}^{T \wedge \rho_{R}}\langle\bar{X}(s)-X(\eta(s)), f(X(\eta(s)), X(\eta(q s)), r(\eta(s)))\rangle \mathrm{d} s \\
& +2 \int_{0}^{T \wedge \rho_{R}}\langle\bar{X}(s), g(X(\eta(s)), X(\eta(q s)), r(\eta(s)))\rangle \mathrm{d} w(s) .
\end{aligned}
$$

For any $x, y \in \mathbb{R}^{n},|x| \leq R,|y| \leq R$, by the local Lipschitz condition (H1), we obtain

$$
\begin{aligned}
|f(x, y, i)|^{2} & \leq 2\left(|f(x, y, i)-f(0,0, i)|^{2}+|f(0,0, i)|^{2}\right) \\
& \leq 2\left(C_{R}|x|^{2}+C_{R}|y|^{2}+|f(0,0, i)|^{2}\right) \\
& \leq C(R)\left(1+|x|^{2}+|y|^{2}\right) \leq C(R)
\end{aligned}
$$

where $C(R)=2 C_{R} \vee|f(0,0, i)|^{2} \vee|g(0,0, i)|^{2}$. Similarly,

$$
|g(x, y, i)|^{2} \leq C(R)\left(1+|x|^{2}+|y|^{2}\right) \leq C(R) .
$$

Substituting (H2) into (3.13) and using (3.14) and (3.15), one may compute

$$
\begin{aligned}
\mathbb{E}\left|\bar{X}\left(T \wedge \rho_{R}\right)\right|^{2} \leq & \mathbb{E}|\bar{X}(0)|^{2}+\mathbb{E} \int_{0}^{T \wedge \rho_{R}}\left[\bar{a}|X(\eta(q s))|^{\alpha+2}-a|X(\eta(s))|^{\alpha+2}\right. \\
& -a_{0}|X(\eta(s))|^{2}+b|X(\eta(s))|^{\alpha+2}+\bar{b}|X(\eta(q s))|^{\alpha+2} \\
& \left.+b_{0}|X(\eta(s))|^{2}\right] \mathrm{d} s+2 C(R) \mathbb{E} \int_{0}^{T \wedge \rho_{R}}|\bar{X}(s)-X(\eta(s))| \mathrm{d} s,
\end{aligned}
$$


noting $\int_{0}^{T \wedge \rho_{R}}\langle\bar{X}(s), g(X(\eta(s)), X(\eta(q s)), r(\eta(s)))\rangle \mathrm{d} w(s)$ is local martingale. Noting that

$$
\mathbb{E} \int_{0}^{T \wedge \rho_{R}}|X(\eta(q s))|^{\alpha+2} \mathrm{~d} s \leq \frac{1}{q} \mathbb{E} \int_{0}^{T \wedge \rho_{R}}|X(\eta(s))|^{\alpha+2} \mathrm{~d} s .
$$

Moreover,

$$
\mathbb{E} \int_{0}^{T \wedge \rho_{R}}|\bar{X}(s)-X(\eta(s))| \mathrm{d} s \leq \mathbb{E} \int_{0}^{T \wedge \rho_{R}}|\bar{X}(s)-\hat{X}(\eta(s))| \mathrm{d} s+\mathbb{E} \int_{0}^{T \wedge \rho_{R}}|\hat{X}(\eta(s))-X(\eta(s))| \mathrm{d} s .
$$

Noting that

$$
\begin{aligned}
|\bar{X}(s)-\hat{X}(\eta(s))| \mathbf{1}_{\left[t_{k}, t_{k+1}\right)}(s)= & \mid \int_{t_{k}}^{s} f(X(\eta(h)), X(\eta(q h)), r(\eta(h))) \mathrm{d} h \\
& +\int_{t_{k}}^{s} g(X(\eta(h)), X(\eta(q h)), r(\eta(h))) \mathrm{d} w(h) \mid 1_{\left[t_{k}, t_{k+1}\right)}(s) .
\end{aligned}
$$

By (3.14), (3.15) and $\mathbb{E}|w(h)| \leq\left[\mathbb{E}(w(h))^{2}\right]^{\frac{1}{2}} \leq(\bar{m} \Delta)^{\frac{1}{2}}$, then

$$
\mathbb{E}|\bar{X}(s)-\hat{X}(\eta(s))| \mathbf{1}_{\left[t_{k}, t_{k+1}\right)}(s) \leq C(R) \Delta^{\frac{1}{2}} .
$$

Therefore

$$
\mathbb{E} \int_{0}^{T \wedge \rho_{R}}|\bar{X}(s)-\hat{X}(\eta(s))| \mathrm{d} s \leq C(R, T) \Delta^{\frac{1}{2}} .
$$

By Lemma 3.3, one may get

$$
\mathbb{E} \int_{0}^{T \wedge \rho_{R}}|\hat{X}(\eta(s))-X(\eta(s))| \mathrm{d} s \leq \mathbb{E} \int_{0}^{T}\left|\hat{X}\left(\eta\left(s \wedge \rho_{R}\right)\right)-X\left(\eta\left(s \wedge \rho_{R}\right)\right)\right| \mathrm{d} s \leq C(R, T) \Delta .
$$

Substituting (3.19) and (3.20) into (3.18), yields

$$
\mathbb{E} \int_{0}^{T \wedge \rho_{R}}|\bar{X}(s)-X(\eta(s))| \mathrm{d} s \leq C(R, T) \Delta^{\frac{1}{2}}+C(R, T) \Delta=C(R, T) \Delta^{\frac{1}{2}} .
$$

Substituting for (3.17) and (3.21) into (3.16),noting that $a_{0}>b_{0}, a>b+\frac{1}{q}(\bar{a}+\bar{b})$, yields

$$
\begin{aligned}
\mathbb{E}\left|\bar{X}\left(T \wedge \rho_{R}\right)\right|^{2} \leq & \mathbb{E}|\bar{X}(0)|^{2}+\mathbb{E} \int_{0}^{T \wedge \rho_{R}}\left[\frac{\bar{a}}{q}|X(\eta(s))|^{\alpha+2}-a|X(\eta(s))|^{\alpha+2}-a_{0}|X(\eta(s))|^{2}\right. \\
& \left.+b|X(\eta(s))|^{\alpha+2}+\frac{\bar{b}}{q}|X(\eta(s))|^{\alpha+2}+b_{0}|X(\eta(s))|^{2}\right] \mathrm{d} s+2 C(R) C(R, T) \Delta^{\frac{1}{2}} \\
\leq & \mathbb{E}|\bar{X}(0)|^{2}-\left(a_{0}-b_{0}\right) \mathbb{E} \int_{0}^{T \wedge \rho_{R}}|X(\eta(s))|^{2} \mathrm{~d} s \\
& -\left(a-b-\frac{\bar{a}+\bar{b}}{q}\right) \mathbb{E} \int_{0}^{T \wedge \rho_{R}}|X(\eta(s))|^{\alpha} \mathrm{d} s+2 C(R) C(R, T) \Delta^{\frac{1}{2}} \\
\leq & \mathbb{E}|\bar{X}(0)|^{2}+C(R, T) \Delta^{\frac{1}{2}} .
\end{aligned}
$$

If $\inf \{t>0:|\bar{X}(t)| \geq R\} \leq \inf \{t>0:|X(\eta(t))|>R\}$, then

$$
\left|\bar{X}\left(\rho_{R}\right)\right|^{2}=R^{2} .
$$

If $\inf \{t>0:|\bar{X}(t)| \geq R\}>\inf \{t>0:|X(\eta(t))|>R\}$, then $\rho_{R}$ is a grid point, and $\left|X\left(\rho_{R}\right)\right|^{2}>$ $R^{2},\left|X\left(\left[q \rho_{R}\right]\right)\right|^{2} \leq R^{2}$. By lemma 3.3, we have

$$
\bar{X}\left(\rho_{R}\right)=\hat{X}\left(\rho_{R}\right)=X\left(\rho_{R}\right)-f\left(X\left(\rho_{R}\right), X\left(\left[q \rho_{R}\right], r\left(\rho_{R}\right)\right) \Delta+f\left(X_{0}, X_{0}, r_{0}\right) \Delta .\right.
$$

Therefore by (H2)

$$
\begin{aligned}
\left|\bar{X}\left(\rho_{R}\right)\right|^{2} \geq & \frac{1}{2}\left|X\left(\rho_{R}\right)\right|^{2}-\left\langle X\left(\rho_{R}\right), f\left(X\left(\rho_{R}\right), X\left(\left[q \rho_{R}\right]\right), r\left(\rho_{R}\right)\right) \Delta\right\rangle \\
& +\frac{1}{2}\left|f\left(X\left(\rho_{R}\right), X\left(\left[q \rho_{R}\right]\right), r\left(\rho_{R}\right)\right) \Delta\right|^{2}-\left|f\left(X_{0}, X_{0}, r_{0}\right) \Delta\right|^{2} \\
\geq & \frac{1}{2}\left|X\left(\rho_{R}\right)\right|^{2}-\left\langle X\left(\rho_{R}\right), f\left(X\left(\rho_{R}\right), X\left(\left[q \rho_{R}\right], r\left(\rho_{R}\right)\right)\right) \Delta\right\rangle-\left|f\left(X_{0}, X_{0}, r_{0}\right) \Delta\right|^{2} \\
\geq & \frac{1}{2}\left|X\left(\rho_{R}\right)\right|^{2}+\frac{a_{0}}{2}\left|X\left(\rho_{R}\right)\right|^{2} \Delta-\left|f\left(X_{0}, X_{0}, r_{0}\right) \Delta\right|^{2}-\frac{\bar{a}}{2}\left|X\left(\left[q \rho_{R}\right]\right)\right|^{\alpha+2} \Delta+\frac{a}{2}\left|X\left(\rho_{R}\right)\right|^{\alpha+2} \Delta,
\end{aligned}
$$


and

$$
\begin{aligned}
\mathbb{E}\left|\bar{X}\left(\rho_{R}\right)\right|^{2} & >\frac{1}{2} R^{2}+\frac{a_{0}}{2} R^{2} \Delta-\frac{\bar{a}}{2} R^{\alpha+2} \Delta+\frac{a}{2} R^{\alpha+2} \Delta-\left|f\left(X_{0}, X_{0}, r_{0}\right)\right|^{2} \Delta^{2} \\
& =\left(\frac{1}{2}+\frac{a_{0}}{2} \Delta\right) R^{2}+\left(\frac{a}{2}-\frac{\bar{a}}{2}\right) R^{\alpha+2} \Delta-\left|f\left(X_{0}, X_{0}, r_{0}\right)\right|^{2} \Delta^{2} \geq c_{1} R^{2}-c_{2} \Delta,
\end{aligned}
$$

where $c_{1}$ and $c_{2}$ are positive constants. Therefore according to (3.23) and (3.24), we have

$$
\mathbb{E}\left|\bar{X}\left(T \wedge \rho_{R}\right)\right|^{2} \geq \mathbb{E}\left[\left|\bar{X}\left(\rho_{R}\right)\right|^{2} 1_{\left\{\rho_{R} \leq T\right\}}\right] \geq P\left(\rho_{R} \leq T\right)\left(c_{1} R^{2}-c_{2} \Delta\right),
$$

which implies that

$$
\mathbb{P}\left(\rho_{R} \leq T\right) \leq \frac{\mathbb{E}\left|\bar{X}\left(T \wedge \rho_{R}\right)\right|^{2}}{c_{1} R^{2}-c_{2} \Delta} \leq \frac{\mathbb{E}|\bar{X}(0)|^{2}+C(R, T) \Delta^{\frac{1}{2}}}{c_{1} R^{2}-c_{2} \Delta},
$$

where the second inequality uses (3.22). For any given $\varepsilon>0$, choose sufficiently large $R_{0}$ such that for any $R \geq R_{0}$

$$
\frac{\mathbb{E}|\bar{X}(0)|^{2}}{c_{1} R^{2}-c_{2} \Delta} \leq \frac{\varepsilon}{2}
$$

Choose sufficiently small $\Delta^{*}$, such that for any $\Delta<\Delta^{*}$

$$
\frac{C(R, T) \Delta^{\frac{1}{2}}}{c_{1} R^{2}-c_{2} \Delta} \leq \frac{\varepsilon}{2}
$$

which implies that $P\left(\rho_{R} \leq T\right) \leq \varepsilon$ as required.

Lemma 3.5 Let (H1) hold. Then for every $t \in\left[0, T \wedge \rho_{R}\right]$,

$$
\begin{aligned}
& \mathbb{E} \int_{0}^{t \wedge \rho_{R}}|f(X(\eta(s)), X(\eta(q s)), r(\eta(s)))-f(X(\eta(s)), X(\eta(q s)), r(s))|^{2} \mathrm{~d} s \leq 8 C_{R}(1+2 C) C_{0} T \Delta, \\
& \mathbb{E} \int_{0}^{t \wedge \rho_{R}}|g(X(\eta(s)), X(\eta(q s)), r(\eta(s)))-g(X(\eta(s)), X(\eta(q s)), r(s))|^{2} \mathrm{~d} s \leq 8 C_{R}(1+2 C) C_{0} T \Delta,
\end{aligned}
$$

where $C_{0}$ is a positive constant dependent on $\max _{0 \leq i \leq N}\left(-\gamma_{i i}\right)$, but independent of $\Delta$.

Proof For $t \in\left[0, T \wedge \rho_{R}\right]$, it is easy to see that

$$
\begin{aligned}
& \mathbb{E} \int_{0}^{t \wedge \rho_{R}}|f(X(\eta(s)), X(\eta(q s)), r(\eta(s)))-f(X(\eta(s)), X(\eta(q s)), r(s))|^{2} \mathrm{~d} s \\
= & \sum_{k=0}^{M-1} \mathbb{E} \int_{t_{k}}^{t_{k+1} \wedge \rho_{R}}|f(X(\eta(s)), X(\eta(q s)), r(\eta(s)))-f(X(\eta(s)), X(\eta(q s)), r(s))|^{2} \mathrm{~d} s .
\end{aligned}
$$

Noting that for $s \in\left[t_{k}, t_{k+1} \wedge \sigma_{R}\right), r(\eta(s))=r\left(t_{k}\right), X(\eta(s))=X\left(t_{k}\right), X(\eta(q s))=X\left(t_{[q k]}\right)$ and $I_{\left\{r(s) \neq r\left(t_{k}\right)\right\}}$ is conditionally independent with respect to the $\sigma$-algebra generated by $r\left(t_{k}\right)$, by this and Lemma 3.2, we may compute

$$
\begin{aligned}
& \mathbb{E} \int_{t_{k}}^{t_{k+1} \wedge \rho_{R}}|f(X(\eta(s)), X(\eta(q s)), r(\eta(s)))-f(X(\eta(s)), X(\eta(q s)), r(s))|^{2} \mathrm{~d} s \\
\leq & 2 \mathbb{E} \int_{t_{k}}^{t_{k+1} \wedge \rho_{R}}\left|f\left(X\left(t_{k}\right), X\left(t_{[q k]}\right), r\left(t_{k}\right)\right)-f\left(X\left(t_{k}\right), X\left(t_{[q k]}\right), r(s)\right)\right|^{2} I_{\left\{r(s) \neq r\left(t_{k}\right)\right\}} \mathrm{d} s \\
\leq & 4 \mathbb{E} \int_{t_{k}}^{t_{k+1} \wedge \rho_{R}}\left[\left|f\left(X\left(t_{k}\right), X\left(t_{[q k]}\right), r\left(t_{k}\right)\right)\right|^{2}+\left|f\left(X\left(t_{k}\right), X\left(t_{[q k]}\right), r(s)\right)\right|^{2}\right] I_{\left\{r(s) \neq r\left(t_{k}\right)\right\}} \mathrm{d} s \\
\leq & 8 C_{R} \mathbb{E} \int_{t_{k}}^{t_{k+1} \wedge \rho_{R}}\left(1+\left|X\left(t_{k}\right)\right|^{2}+\left|X\left(t_{[q k]}\right)\right|^{2}\right) I_{\left\{r(s) \neq r\left(t_{k}\right)\right\}} \mathrm{d} s \\
\leq & 8 C_{R} \int_{t_{k}}^{t_{k+1}} \mathbb{E}\left(\mathbb{E}\left[\left(1+\left|X\left(t_{k}\right)\right|^{2}+\left|X\left(t_{[q k]}\right)\right|^{2}\right) I_{\left\{r\left(s \wedge \rho_{R}\right) \neq r\left(t_{k}\right)\right\}} \mid r\left(t_{k}\right)\right]\right) \mathrm{d} s \\
\leq & 8 C_{R} \int_{t_{k}}^{t_{k+1}} \mathbb{E}\left(1+\left|X\left(t_{k}\right)\right|^{2}+\mid X\left(\left.t_{[q k]}\right|^{2}\right) \mathbb{E}\left[I_{\left\{r\left(s \wedge \rho_{R}\right) \neq r\left(t_{k}\right)\right\}} \mid r\left(t_{k}\right)\right] \mathrm{d} s\right. \\
\leq & 8 C_{R}(1+2 C) \int_{t_{k}}^{t_{k+1}} \mathbb{E}\left[I_{\left\{r\left(s \wedge \rho_{R}\right) \neq r\left(t_{k}\right)\right\}} \mid r\left(t_{k}\right)\right] \mathrm{d} s .
\end{aligned}
$$


By the Markov property, we have

$$
\begin{aligned}
\mathbb{E}\left[I_{\left\{r\left(s \wedge \rho_{R}\right) \neq r\left(t_{k}\right)\right\}} \mid r\left(t_{k}\right)\right] & =\sum_{i \in S} I_{\left\{r\left(t_{k}\right)=i\right\}} P\left(r\left(s \wedge \rho_{R}\right) \neq i \mid r\left(t_{k}\right)=i\right) \\
& =\sum_{i \in S} I_{\left\{r\left(t_{k}\right)=i\right\}} \sum_{j \neq i}\left(\gamma_{i j}\left(s \wedge \sigma_{R}-t_{k}\right)+o\left(s \wedge \rho_{R}-t_{k}\right)\right) \\
& =\left(\max _{1 \leq i \leq N}\left(-\gamma_{i i} \Delta+o(\Delta)\right)\right) \sum_{i \in S} I_{\left\{r\left(t_{k}\right)=i\right\}} \leq C_{0} \Delta,
\end{aligned}
$$

where $C_{0}$ is constant independent of $\Delta$. Substituting (3.26) into (3.25), yields

$\mathbb{E} \int_{t_{k}}^{t_{k+1} \wedge \rho_{R}}|f(X(\eta(s)), X(\eta(q s)), r(\eta(s)))-f(X(\eta(s)), X(\eta(q s)), r(s))|^{2} \mathrm{~d} s \leq 8 C_{R}(1+2 C) C_{0} \Delta^{2}$.

The section inequality is proved similarly.

Lemma 3.6 Let (H1), (H2) and (H3) hold with $a_{0}>m b_{0}, a>m b+(\bar{a}+m \bar{b}) / q$, for sufficiently large $R$, there exists a positive constant $C(R, T)$ such that

$$
\mathbb{E}\left[\sup _{0 \leq t \leq T}\left|\bar{X}\left(t \wedge \gamma_{R}\right)-x\left(t \wedge \gamma_{R}\right)\right|^{2}\right] \leq C(R, T) \Delta
$$

where $\gamma_{R}=\nu_{R} \wedge \rho_{R}$.

Proof For any $T_{1} \in[0, T]$, by the Hölder's and the Burkhölder-Davis-Gundy's inequalities

$$
\begin{aligned}
& \mathbb{E}\left[\sup _{0 \leq t \leq T_{1}}\left|\bar{X}\left(t \wedge \gamma_{R}\right)-x\left(t \wedge \gamma_{R}\right)\right|^{2}\right] \\
\leq & T \mathbb{E} \int_{0}^{T_{1} \wedge \gamma_{R}}|f(X(\eta(s)), X(\eta(q s)), r(\eta(s)))-f(x(s), x(q s), r(s))|^{2} \mathrm{~d} s \\
& \left.+4 \mathbb{E} \int_{0}^{T_{1} \wedge \gamma_{R}}|g(X(\eta(s)), X(\eta(q s)), r(\eta(s)))-g(x(s), x(q s), r(s))|^{2} \mathrm{~d} s\right) .
\end{aligned}
$$

By the local Lipschitz condition (H1) and Lemma 3.5, we may compute

$$
\begin{aligned}
& \mathbb{E} \int_{0}^{t \wedge \gamma_{R}}|f(X(\eta(s)), X(\eta(q s)), r(\eta(s)))-f(x(s), x(q s), r(s))|^{2} \mathrm{~d} s \\
\leq \quad & 2 \mathbb{E} \int_{0}^{t \wedge \gamma_{R}}|f(X(\eta(s)), X(\eta(q s)), r(\eta(s)))-f(X(\eta(s)), X(\eta(q s)), r(s))|^{2} \mathrm{~d} s \\
& +2 \mathbb{E} \int_{0}^{t \wedge \gamma_{R}}|f(X(\eta(s)), X(\eta(q s)), r(s))-f(x(s), x(q s), r(s))|^{2} \mathrm{~d} s \\
\leq & 16 C_{R}\left(1+2 C_{1}\right) C_{0} T \Delta+2 \mathbb{E} \int_{0}^{t \wedge \gamma_{R}}|f(X(\eta(s)), X(\eta(q s)), r(s))-f(x(s), x(q s), r(s))|^{2} \mathrm{~d} s .
\end{aligned}
$$

According to (H1) and (3.21), we may compute

$$
\begin{aligned}
& \mathbb{E} \int_{0}^{t \wedge \gamma_{R}}|f(X(\eta(s)), X(\eta(q s)), r(s))-f(x(s), x(q s), r(s))|^{2} \mathrm{~d} s \\
\leq & \left.C_{R} \mathbb{E} \int_{0}^{t \wedge \gamma_{R}}|X(\eta(s))-x(s)|^{2}+|X(\eta(q s))-x(q s)|^{2}\right) \mathrm{d} s \\
\leq & 2 C_{R} \mathbb{E} \int_{0}^{t \wedge \gamma_{R}}|X(\eta(s))-x(s)|^{2} \mathrm{~d} s \\
\leq & 2 C_{R} \mathbb{E} \int_{0}^{t \wedge \gamma_{R}}\left[|X(\eta(s))-\bar{X}(s)|^{2}+|\bar{X}(s)-x(s)|^{2}\right] \mathrm{d} s \\
\leq & 2 C_{R}\left(C(R, T) \Delta+\mathbb{E} \int_{0}^{t \wedge \gamma_{R}}|\bar{X}(s)-x(s)|^{2} \mathrm{~d} s\right) .
\end{aligned}
$$

This, together with (3.27), yields

$$
\begin{aligned}
& \mathbb{E}\left[\sup _{0 \leq t \leq T_{1}}\left|\bar{X}\left(t \wedge \gamma_{R}\right)-x\left(t \wedge \gamma_{R}\right)\right|^{2}\right] \\
\leq & C(R, T) \Delta+4 C_{R}(T+4) \int_{0}^{T_{1}} \mathbb{E}\left[\sup _{0 \leq t \leq s}\left|\bar{X}\left(s \wedge \gamma_{R}\right)-x\left(s \wedge \gamma_{R}\right)\right|^{2}\right] \mathrm{d} s .
\end{aligned}
$$


The Gronwall inequality implies that the conclusion holds.

Theorem 3.7 Let (H1)-(H3)) hold with $a_{0}>m b_{0}, a>m b+(\bar{a}+m \bar{b}) / q$, For any given $T=$ $N \Delta>0, s \in[1,2)$, the backward EM scheme (3.1) has the property

$$
\lim _{\Delta \rightarrow 0} \mathbb{E}|X(T)-x(T)|^{s}=0 .
$$

Proof Denoted by $e(T)=X(T)-x(T)$. Applying the Young's inequality

$$
a^{s} b \leq \frac{\sigma s}{2} b^{2}+\frac{2-s}{2 \sigma^{\frac{2}{2-s}}} b^{\frac{2}{2-s}}, \forall a, b, \sigma>0,
$$

yields

$$
\begin{aligned}
\mathbb{E}|e(T)|^{s}= & \mathbb{E}\left[|e(T)|^{s} \mathbf{1}_{\left\{\nu_{R}>T, \rho_{R}>T\right\}}\right]+\mathbb{E}\left[|e(T)|^{s} \mathbf{1}_{\left\{\nu_{R} \leq T \text { or } \rho_{R} \leq T\right\}}\right] \\
\leq & 2^{s-1}\left[\mathbb{E}\left[|X(T)-\bar{X}(T)|^{s} \mathbf{1}_{\left\{\nu_{R}>T, \rho_{R}>T\right\}}\right]+\mathbb{E}\left[|\bar{X}(T)-x(T)|^{s} \mathbf{1}_{\left\{\nu_{R}>T, \rho_{R}>T\right\}}\right]\right] \\
& +\frac{\sigma s}{2} \mathbb{E}\left[|e(T)|^{2}\right]+\frac{2-s}{2 \sigma^{\frac{2}{2-s}}} \mathbb{P}\left(\nu_{R} \leq T \text { or } \rho_{R} \leq T\right) .
\end{aligned}
$$

The Hölder inequality and Lemma 3.3 implies that

$$
\mathbb{E}\left[|X(T)-\bar{X}(T)|^{s} \mathbf{1}_{\left\{\nu_{R}>T, \rho_{R}>T\right\}}\right] \leq\left(\mathbb{E}\left[|X(T)-\hat{X}(T)|^{p} \mathbf{1}_{\left\{\nu_{R}>T, \rho_{R}>T\right\}}\right]\right)^{\frac{s}{p}} \leq C(p, R) \Delta^{s} .
$$

The Hölder inequality, Lemma 3.6 implies that

$$
\mathbb{E}\left[|\bar{X}(T)-x(T)|^{s} \mathbf{1}_{\left\{\nu_{R}>T, \rho_{R}>T\right\}}\right] \leq\left(\mathbb{E}\left[|\bar{X}(T)-x(T)|^{2} \mathbf{1}_{\left\{\nu_{R}>T, \rho_{R}>T\right\}}\right]\right)^{\frac{s}{2}} \leq C(R, T) \Delta^{\frac{s}{2}} .
$$

For any given $\varepsilon>0$, by the Hölder inequality and Theorem 2.1 and Lemma 3.2, we choose $\sigma>0$ such that

$$
\frac{\sigma s}{2} \mathbb{E}\left[|e(T)|^{2}\right] \leq \sigma s \mathbb{E}\left[|x(T)|^{2}+|X(T)|^{2}\right] \leq \frac{\epsilon}{3} .
$$

By Theorem 2.1, there exists sufficiently large $R_{0}$ such that for $R \geq R_{0}$

$$
\frac{2-s}{2 \sigma^{\frac{s}{2-s}}} \mathbb{P}\left(\nu_{R} \leq T\right) \leq \frac{\epsilon}{3} .
$$

Substituting (3.29)-(3.32) into (3.28), yields

$$
\mathbb{E}|e(T)|^{s} \leq 2^{s-1}\left[C(p, R) \Delta^{s}+C(R, T) \Delta^{\frac{s}{2}}\right]+\frac{2-s}{2 \delta^{\frac{s}{2-s}}} \mathbb{P}\left(\rho_{R} \leq T\right)+\frac{2 \epsilon}{3} .
$$

Applying Lemma 3.4 and choosing sufficiently small $\Delta$ such that

$$
2^{s-1}\left[C(p, R) \Delta^{s}+C(R, T) \Delta^{\frac{s}{2}}\right]+\frac{2-s}{2 \delta^{\frac{s}{2-s}}} \mathbb{P}\left(\rho_{R} \leq T\right) \leq \frac{\epsilon}{3} .
$$

The conclusion holds.

\section{Exponential Stability of numerical solution}

In the section, we shall prove that the backward EM method can preserve the almost surely exponential stability of the true solution of highly nonlinear stochastic pantograph differential equation.

(H4)(The polynomial growth conditions) For any $x \in \mathbb{R}^{d}$, there exist positive constants $\alpha, a, \bar{a}$, $\tilde{a}, b, \bar{b}, \tilde{b}$ such that

$$
\langle x(s), f(x(s), x(q s), i)\rangle \leq-a|x(s)|^{\alpha+2}+\bar{a} \mathrm{e}^{-(1-q) \varepsilon s}|x(q s)|^{\alpha+2}-a_{0}|x(s)|^{2},
$$




$$
|g(x(s), x(q s), i)|^{2} \leq b|x(s)|^{\alpha+2}+\bar{b} \mathrm{e}^{-(1-q) \varepsilon s}|x(q s)|^{\alpha+2}+b_{0}|x(s)|^{2} .
$$

Under (H4), Eq.(2.1) has a unique global solution and the solution is almost surely exponentially stable(see[26]).

Theorem 4.1Let (H1),(H3) and (H4) hold with $\alpha \geq \beta \vee \gamma, 2 a_{0}>b_{0}, 2 a>2 \bar{a}(1+1 / q)+b+\bar{b} / q$. Then for any initial data $\xi$, the solution $x(t)$ is pth moment and almost surely exponentially stable, i.e.

$$
\lim \sup _{t \rightarrow \infty} \frac{1}{t} \log |x(t)| \leq-\frac{\varepsilon}{2}
$$

where $\varepsilon \leq 2 a_{0}-b_{0}$.

The proof is similar to Theorem 3 in Zhou[26].

Theorem 4.2 Let (H1),(H3) and (H4) hold with $2 a_{0}>m b_{0}, 2 a>m b+(2 \bar{a}+m \bar{b}) / q$, there exists a sufficiently small $\Delta^{*} \in(0,1)$ such that the approximate solution $\left\{X_{k}\right\}$ defined by (3.1) satisfies

$$
\lim \sup _{k \rightarrow \infty} \frac{1}{k \Delta} \log \left|X_{k}\right| \leq-\frac{\varepsilon}{2} \text { a.s. }
$$

where $\varepsilon<2 a_{0}-m b_{0}$.

The proof is similar to Theorem 7 in Zhou[26].

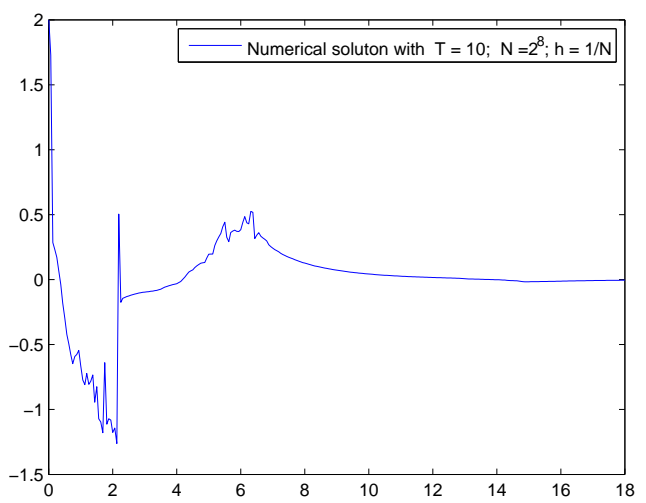

Figure 1: Computer simulation of the path $x(t)$ for Eq.(4.1) with step size $\Delta=2^{-8}$.

In the following, we shall consider a stochastic pantograph equation to illustrate our theory established in the previous sections.

Example 4.3 Consider the following nonlinear scalar stochastic pantograph equation

$$
\mathrm{d} x(t)=\left[-a(r(t)) x(t)-b(r(t)) x^{5}(t)+x^{5}(q t)\right] \mathrm{d} t+\left[c(r(t)) x^{2}(t) x(q t)+x^{3}(t)\right] \mathrm{d} w(t),
$$

with the initial condition $x_{0}=1, q=0.5$, where $w(t)$ is scalar Brownian motion.

Define $f(x, y, i)=-a_{i} x-b_{i} x^{5}+y^{5}, g(x, y)=c_{i} x^{2} y+x^{3}$. Compute

$$
\begin{aligned}
f\left(x_{1}, y, i\right)-f\left(x_{2}, y, i\right) & \leq-a_{i}\left(x_{1}-x_{2}\right)-b_{i}\left(x_{1}^{5}-x_{2}^{5}\right) \\
& \leq-a_{i}\left(x_{1}-x_{2}\right)-b_{i}\left(x_{1}-x_{2}\right)\left(x_{1}^{4}+x_{1}^{3} x_{2}+x_{2}^{2} x_{2}^{2}+x_{1} x_{2}^{3}+x_{2}^{4}\right) \\
& \leq-\left(x_{1}-x_{2}\right)\left[a_{i}+b_{i}\left(x_{1}^{4}+x_{1}^{3} x_{2}+x_{2}^{2} x_{2}^{2}+x_{1} x_{2}^{3}+x_{2}^{4}\right)\right] .
\end{aligned}
$$


Noting that $a^{2}+b^{2} \geq(a+b)^{2} / 2$, compute

$$
\begin{aligned}
x_{1}^{4}+x_{1}^{3} x_{2}+x_{2}^{2} x_{2}^{2}+x_{1} x_{2}^{3}+x_{2}^{4} & \geq\left(x_{1}^{4}+x_{2}^{4}\right)+x_{1} x_{2}\left(x_{1}^{2}+x_{2}^{2}\right)+\left(x_{1} x_{2}\right)^{2} \\
& \geq \frac{\left(x_{1}^{2}+x_{2}^{2}\right)^{2}}{2}+x_{1} x_{2}\left(x_{1}^{2}+x_{2}^{2}\right)+\left(x_{1} x_{2}\right)^{2} \\
& \geq \frac{\left(x_{1}^{2}+x_{2}^{2}\right)^{2}}{4}+x_{1} x_{2}\left(x_{1}^{2}+x_{2}^{2}\right)+\left(x_{1} x_{2}\right)^{2} \\
& \geq\left[\frac{x_{1}^{2}+x_{2}^{2}}{2}+x_{1} x_{2}\right]^{2},
\end{aligned}
$$

which implies

$$
\left\langle x_{1}-x_{2}, f\left(x_{1}, y, i\right)-f\left(x_{2}, y, i\right)\right\rangle \leq-a_{i}\left(x_{1}-x_{2}\right)^{2} .
$$

This implies that $f(x, y, i)$ satisfies the one-sided Lipschitz condition (H3). By using the inequality $a^{p} b^{q} \leq \frac{p}{p+q} a^{p+q}+\frac{q}{p+q} b^{p+q}$, it is easy to compute

$2\langle x, f(x, y, i)\rangle \leq-2 a_{i} x^{2}-2 b_{i} x^{6}+2 y^{5} x \leq-2 a_{i} x^{2}-2 b_{i} x^{6}+5 y^{6} / 3+x^{6} / 3 \leq-2 a_{i} x^{2}+5 y^{6} / 36-\left(2 b_{i}-1 / 3\right) x^{6}$,

$$
|g(x, y, i)|^{2}=\left(c_{i} x^{2} y+x^{3}\right)^{2} \leq 2 c_{i}^{2} x^{4} y^{2}+2 x^{6} \leq 2 c_{i}^{2} y^{6} / 3+\left(4 c_{i}^{2} / 3+2\right) x^{6} .
$$

Applying Theorem 2.1 with $\alpha=4, a_{0}=2 \min _{1 \leq i \leq N} a_{i}, \bar{a}=5 / 3, a=\min _{1 \leq i \leq N} b_{i}-1 / 3, b_{0}=0, \bar{b}=$ $2 \min _{1 \leq i \leq N} c_{i}^{2} / 3, b=4 \min _{1 \leq i \leq N} c_{i}^{2} / 3+2$. Clearly, $a_{0}>\bar{b}_{0}, a>b+(\bar{a}+\bar{b}) / q$ with $p=2$, then Eq.(4.3) has a unique global solution. For example, choose $a_{1}=0.5, b_{1}=4, c_{1}=0.5$ and $a_{2}=0.2, b_{2}=3, c_{2}=$ 0.2 , then $a_{0}=0.4, \bar{a}=5 / 3, a=17 / 3, b_{0}, \bar{b}=2 / 75, b=154 / 75$, clearly, $a_{0}>b_{0}, a>b+(\bar{a}+\bar{b}) / q$ holds, so Eq.(4.3) has a unique global solution.

Moreover, the discrete backward EM solution is defined by

$$
\begin{aligned}
X\left(t_{k+1}\right)= & X\left(t_{k}\right)+\left[-0.5 X\left(t_{k+1}\right)-4 X^{5}\left(t_{k+1}\right)+X^{5}\left(t_{[q(k+1)]}\right)\right] \Delta \\
& \left.+\left[0.5 X^{2}\left(t_{k}\right) X\left(t_{[q k]}\right)\right)+X^{3}\left(t_{k}\right)\right] \mathrm{d} \Delta w_{t_{k}} .
\end{aligned}
$$

Obviously, $a>b+\frac{1}{q}(\bar{a}+\bar{b})$, on the basis of Theorem 4.4 the backward approximate solution converges to the true solution. In Figs.1, we plot the Euler-Maruyama method of Eq.(4.3) for step size $\Delta=1 / 256$. The figure illustrates that the numerical solution converges strongly to zero.

\section{References}

[1] Appleby J.A.D.: Decay and growth rates of solutions of scalar stochastic delay differential equations with unbounded delay and state dependent noise. Stoch. Dyn.5, 133-147(2005)

[2] Baker C.T.H., Buckwar E.: Continuous $\theta$-methods for the stochastic pantograph equation. Electron Trans. Number. Anal.11, 131-151(2000)

[3] Balasubramaniam P., Ntouyas S.: Controllability for neutral stochastic functional differential inclusions with infinite delay in abstract space. J. Math. Anal. Appl. 324, 161-176(2006)

[4] Fan Z., Liu M., Cao W.: Existence and uniqueness of the solutions and convergence of semiimplicit Euler methods for stochastic pantograph equations. J. Math. Anal. Appl.325, 1142-1159(2007) 
[5] Fan Z., Song M., Liu M.: The $\alpha$ th moment stability for the pantograph equation. J. Comput. Appl. Math. 233, 109-120(2009)

[6] Hassane B., Fu X.: Controllability of neutral functional differential equations with infinite delay. Acta Math. Sci. 31, 73-80(2011)

[7] Hu Y., Wu F.: Stochastic Kolmogorov-type population dynamics with infinite distributed delays. Acta Appl. Math. 11,1407-1428(2010)

[8] Huang C., Cao J.: Almost sure exponential stability of stochastic cellular neural networks with unbounded distributed delays. Neurocomputing 72, 3352-3356(2009)

[9] Li R., Liu M., Pang W.: Convergence of numerical solutions to stochastic pantograph equations with Markovian switching. Appl. Math. Comput. 215, 414-422 (2009)

[10] Li X., Fu X.: Stability analysis of stochastic functional differential equations with infinite delay and its application to recurrent neural networks. J. Comput. Appl. Math. 234, 407-417(2010)

[11] Mao X.: Exponential Stability of Stochastic Differential Equation. Dekker, NewYork(1994)

[12] Mao X., Shen Y.: Alison Gray, Almost sure exponential stability of backward Euler-Maruyama discretization for hybrid stochastic differential equation. J. Comput. Appl. Math.235, 1213-1226 (2011)

[13] Mao X., Szpruch L.: Strong convergence rates for backward Euler-maruyama method for non-linear dissipative-type stochastic differential equations with super-linear diffusion coefcients, Stochastic. an Inter. J. Prob. Stoch. Proc.56, 1-28(2012)

[14] Mao X., Szpruch L.: Strong convergence and stability of implicit numerical methods for stochastic differential equations with non-globally lipschitz continuous coeffcients. J. Comput. Appl. Math. 238, 14-28(2012)

[15] Meng X., Tian M., Hu S.: Stability analysis of stochastic recurrent neural networks with unbounded time-varying delays. Neurocomputing 72, 355-367(2011)

[16] Meng X., Hu S., Wu P.: Pathwise estimation of stochastic differential equations with unbounded delay its application to stochastic pantograph equations. Acta Appl. Math.113, 231-246(2011)

[17] Milošević M., Jovanović M.: A Taylor polynomial approach in approximations of solution to pantograph stochastic differential equations with Markovian switching. Math. Comput. Mod. 53,280$293(2011)$

[18] Ockendon J.R., Tayler A.B.: The dynamics of a current collection system for an electric locomotive. Proc. R. Soc. Lond. Ser. A 322, 447-468(1971)

[19] Szpruch L., Mao X., Higham D.J., Pan J.: Numerical simulation of a strongly nonlinear ait-sahaliatype interest rate model, BIT Numer. Math.51, 405-425(2011) 
[20] Wei F., Wang K.: The existence and uniqueness of the solution for stochastic functional differential equations with infinite delay. J. Math. Anal. Appl. 331, 516-531(2007)

[21] Wu F., Hu S., Mao X.: Razumikhin-type theorem for neutral stochastic functional differential equations with unbounded delay. Acta Math. Sci. 31, 1245-1258(2011)

[22] Zhou S., Xue M.: Exponential stability of highly nonlinear stochastic pantograph equation and numerical approximation. Acta Mathematisa Scientia 4(2014) 1-17

[23] Zhou S., Xie S.: Almost surely exponential stability of the backward Euler-Maruyama discretization for highly nonlinear stochastic functional differential equation. Applied Mathematics and Computation, Volume 236, 1 June 2014, Pages 150-160.

[24] Zhou S., Wang Z., Feng D.: Stochastic functional differential equations with infinite delay. J. Math. Anal. Appl. 357, 416-426(2009)

[25] Zhou S., Hu S., Cen L.: Stochastic Kolmogorov-type system with infinite delay. Appl. Math. Comput. 218, 207-218(2011)

[26] Zhou S.: Almost surely exponential stability of numerical solutions for stochastic pantograph equations, Abstract and Applied Analysis Volume 2014, Article ID 751209, 10 pages http://dx.doi.org/10.1155/2014/751209. 